Financial Statistical Journal (2018) Volume 1 doi:10.24294/fsj.v1i3.687

\title{
Prediction Intervals in the ARFIMA Model Using Bootstrap G
}

\section{Glaura C. Franco $^{1 *}$, Gustavo C. Lana ${ }^{1}$, Valderio A. Reisen ${ }^{2}$}

${ }^{1}$ Department of Statistics, UFMG, MG, Brazil.

${ }^{2}$ Department of Statistics, UFES, ES, Brazil and Laboratoire des Signaux et Systèmes (L2S),

CNRS-CentraleSupèlec-UniversitéParis-Sud, Gif sur Yvette, France

Corresponding author: Glaura C. Franco. Address: Departamento de Estatística - UFMG - Av. AntonioCarlos , 6627 -

Belo Horizonte - MG - Brazil - CEP: 31270-901.

\section{ABSTRACT}

This paper presents a bootstrap resampling scheme to build prediction intervals for future values in fractionally autoregressive moving average (ARFIMA) models. Standard techniques to calculate forecast intervals rely on the assumption of normality of the data and do not take into account the uncertainty associated with parameter estimation. Bootstrap procedures, as nonparametric methods, can overcome these diculties. In this paper, we test two bootstrap prediction intervals based on the nonparametric bootstrap in the residuals of the ARFIMA model. In this paper, two bootstrap prediction intervals are proposed based on the nonparametric bootstrap in the residuals of the ARFIMA model. The rst one is the well known percentile bootstrap, (Thombs and Schucany, 1990; Pascual et al., 2004), never used for ARFIMA models to the knowledge of the authors. For the second approach, the intervals are calculated using the quantiles of the empirical distribution of the bootstrap prediction errors (Masarotto, 1990; Bisaglia e Grigoletto, 2001). The intervals are compared, through a Monte Carlo experiment, to the asymptotic interval, under Gaussian and non-Gaussian error distributions. The results show that the bootstrap intervals present coverage rates closer to the nominal level assumed, when compared to the asymptotic standard method. An application to real data of temperature in New York city is also presented to illustrate the procedures.

Keywords: Bootstrap; Long-memory; Forecasting; Prediction Intervals

\section{Introduction}

The applicability of the bootstrap in forecasting and construction of prediction intervals in different classes of models has been already addressed by many authors, especially for autoregressive moving average (ARMA) models. In Thombs and Schucany (1990) and Pascual et al. (2004), the bootstrap is used to construct an empirical distribution for the future values conditional on the past $\mathrm{p}$ observations, where $\mathrm{p}$ is the number of auto regressive coefficients. Meanwhile, Masarotto (1990) and Pan and Politis (2016) construct, using the bootstrap, an empirical distribution of the prediction errors. Other works on this subject also include Wall and Stoffer (2002), Pfeffermann and Tiller (2005), and Rodriguez and Ruiz(2010). The bootstrap can incorporate the variability due to parameter estimation without assuming any particular distribution for the errors, which can be valuable in real time series, often characterized by short data set and no information about the underlying distribution.

The works referred above employ bootstrap methods to classical procedures conditional on some observations of the original series, but if the time series presents the long memory characteristic, more specific models are needed. One of the most used methodology to deal with this kind of long memory data is the ARFIMA model (Hosking, 1981; Granger and Joyeux, 1980), a generalization of the ARIMA model from Box and Jenkins (1976). In the frequency domain, this feature implies that the spectrum is proportional to $|\omega|^{-2 \mathrm{~d}}$ as the frequency $\omega$ approaches zero, whered is the memory parameter, while in the time domain the auto correlations present a hyperbolic decay. Although there is a rising interest in the ARFIMA model, little has been done concerning the construction of prediction intervals using

Copyright (C) 2018 Glaura C. Franco et al.

doi: $10.24294 /$ fsj.v1i3.687

EnPress Publisher LLC.This work is licensed under the Creative Commons Attribution-NonCommercial 4.0 International License (CC BY-NC 4.0). http://creativecommons.org/licenses/ by/4.0/ 
bootstrap methods. Among them, Rupasinghe and Samaranayake (2012), Rupasinghe et al. (2014) and Bisaglia and Grigoletto (2001).

Thus, the main goal of this paper is to construct prediction intervals in ARFIMA models using the bootstrap. Prediction intervals can be far more informative than a point estimate of the future value, as they can provide a measure of uncertainty of this estimate around the true future value.The ARFIMA model can be written in an infinite autoregressive form, therefore the predictions can be built exactly in the same way as in the ARIMA procedure. The prediction errors, in the standard Box and Jenkins (1976) approach, are assumed to be Gaussian and asymptotic intervals are obtained conditional on parameter estimates. For not taking into account the variability due to parameter estimation, these intervals may have coverage rates which are different from the nominal ones, especially when the errors are non Gaussian. There is some literature on forecasting in long memory models, as the works of Boutahar (2007), Bhansali and Kokoszka (2002) and Crato and Ray (1996),among others.

There are many procedures for bootstrapping in long memory processes and a comparison of some of these methods can be found in Franco and Reisen (2007). In this work, the procedure adopted is the nonparametric bootstrap, obtained resampling the residuals of the fitted model, which are generally uncorrelated if there is not order misspecification.

In this paper, the estimators for the long memory parameter are based on the parametric method, using the maximum likelihood procedure (Fox and Taqqu, 1986), and on the semiparametric approach, calculating a regression equation with the periodogram function (Geweke and Porter-Hudark, 1983).

The paper is organized as follows: In Section 2 the ARFIMA modelis described along with forecasts for future values. The bootstrap procedure and presidiction intervals used in this article are discussed in Section 3 . Section 4 shows some simulation results and Section 5 presents an application on a real time series. Section 6 concludes the work.

\section{Long memory and forecasting}

Let $\left\{X_{t}, t \in \mathbb{Z}\right\}$ be a stationary fractionally integrated ARMA model (ARFIMA), which satisfies

$$
\mathrm{X}_{\mathrm{t}}-\mu=\phi(\mathrm{B})^{-1}(1-\mathrm{B})^{-\mathrm{d}} \theta(\mathrm{B}) \epsilon_{\mathrm{t}}
$$

where $\phi(\mathrm{B})$ and $\theta(\mathrm{B})$ are polynomials of order $p$ and $q$, respectively, with all roots outside the unit circleand $B$ is the backshift operator. $\epsilon_{\mathrm{t}}$ is a sequence ofzero-mean independent random variables with common distributionfunction $\mathrm{F}_{\epsilon}$ and finite variance $\sigma_{\epsilon}^{2},(1-B)^{d}$ is the fractional differencing operator and $\mu$ is a Constant, dis theparameter that holds the memory of the process and when $d \in(-0.5,0.5)$ the ARFIMA $(p, d, q)$ process is said to be invertibleand stationary. Besides, for $d=0, d \in(-0.5,0)$ or $d \in(0,0.5)$, the process is said to be short-memory,intermediate-memory or long-memory, respectively. Stationary longmemory processes are defined by the behavior of the spectraldensity function, which becomes unbounded at zero frequency. Inthe time domain, this means that the autocovariances are notabsolutely summable. In the intermediate-memory region, the autocovariances are absolutely summable and, consequently, the spectral density is bounded. A more detailed description of ARFIMA models can be found in Doukham et al.(2002).

For a stationary ARFIMA process, the spectral density of $\mathrm{X}_{\mathrm{t}}$ is given by

$$
f_{X}(\omega)=f_{U}(\omega)[2 \sin (\omega / 2)]^{-2 d}, \quad \omega \in[-\pi, \pi]
$$

where the function $f_{U}(\omega)$ is the spectral density of the $\operatorname{ARMA}(p, q)$ process, $U_{t}=(1-B)^{d}\left(X_{t}-\mu\right)$. $f_{X}(\omega)$ satisfies the property $f_{X}(\omega) \sim|\omega|^{-2 d}$, as $\omega \rightarrow 0$.

Now, let $\mathrm{X}_{\mathrm{t}}$ be a time series with length $n$ satisfying Equation 1. The usual unbiased but inconsistent estimator of the spectral function is the periodogram function, $I(\omega)=(2 \pi n)^{-1}\left|\sum_{i=1}^{n} X_{t} e^{i \omega t}\right|^{2}$.

As previously mentioned, the estimators for the long memory parameter d considered in this work are the parametric estimator provided by Fox and Taqqu (1986)(FT) and the semiparametric estimator proposed by Geweke and Porter-Hudark (1983) (GPH). The FT estimator estimates all parameters in the model simultaneously, white the GPH estimator only estimates the parameter $\mathrm{d}$. In the case of the GPH estimator, after estimating $\mathrm{d}$ and differencing the series accordingly, the short memory parameters were estimated in this work by maximum likelihood. 
Based on the Gaussian assumption, the estimator FT is obtained byusing all harmonic frequencies and it is calculated by minimizing the approximate Gaussian log-likelihood

$$
\mathcal{L}(\gamma, \mathrm{x})=\frac{1}{2 \mathrm{n}} \sum_{\mathrm{j}=1}^{\mathrm{n}}\left[\ln \mathrm{f}_{\mathrm{x}}\left(\omega_{\mathrm{j}}\right)+\frac{\mathrm{I}\left(\omega_{\mathrm{j}}\right)}{\mathrm{f}_{\mathrm{x}}\left(\omega_{\mathrm{j}}\right)}\right],
$$

where $\gamma=\left(\mathrm{d}, \phi_{1}, \ldots, \phi_{\mathrm{p}}, \theta_{1}, \ldots, \theta_{\mathrm{q}}, \sigma_{\epsilon}^{2}\right)$ denotes the vector of unknown parameters and $\omega_{j}=2 \pi j / n, j=1, \ldots, n$ are the Fourier frequencies.Fox and Taqqu (1986) show that for strongly dependent Gaussian processes the FT estimator, $\hat{\beta}_{\mathrm{FT}}$, is consistent and $\sqrt{n}\left(\widehat{\beta}_{\mathrm{FT}}-\beta\right)$ converges in distribution to $\mathrm{N}\left(0, \mathrm{~V}^{-1}\right)$, where Vis a matrix with elements $V_{\text {ij }}$ given by

$$
V_{i j}=\frac{1}{4 \pi} \int_{-\pi}^{\pi}\left[\frac{\partial \log f_{\beta, \sigma^{2}}(\omega)}{\partial \beta_{i}} \frac{\partial \log f_{\beta, \sigma^{2}}(\omega)}{\partial \beta_{j}}\right] d \omega,
$$

where $\beta=\left(\mathrm{d}, \phi_{1}, \ldots, \phi_{p}, \theta_{1}, \ldots, \theta_{q}, \sigma_{\epsilon}^{2}\right)$ is the parameter vector, $\beta_{i}$ are the elements of $\beta$ and $\mathrm{f}_{\beta, \sigma^{2}}$ is the spectral function of $\mathrm{X}_{\mathrm{t}}$ indexed by parameters $\beta$ and $\sigma^{2}$.

The GPH estimator is obtained by taking the logarithm of the spectral density (2),

$$
\ln f_{x}(\omega)=\ln f_{u}(0)-d \ln [2 \sin (\omega / 2)]^{2}+\ln \left[\frac{f_{u}(\omega)}{f_{u}(0)}\right] .
$$

The estimate of $d$ can be obtained by ordinary least square,substituting the spectral density in (3) by the periodogram function, $\mathrm{I}(\omega)$, as the dependent variable, andln $(2 \sin (\omega / 2))^{2}$ as the independent variable.

Under some conditions, Hurvich et al. (1998)proved that the GPH method is a consistent estimator for the memory parameter and asymptotically normal for Gaussian time series processes. Theauthors established that the optimal bandwidth $\mathrm{g}(\mathrm{n})$ in the regression equation is of order $\mathrm{o}\left(\mathrm{n}^{4 / 5}\right)$ and $(\mathrm{m})^{1 / 2}\left(\mathrm{~d}_{\mathrm{GPH}}-\mathrm{d}\right) \stackrel{\mathrm{d}}{\rightarrow} \mathrm{N}\left(0, \frac{\pi^{2}}{24}\right)$.

An important issue in time series analysis is to predict future values, based on the fitted model. Assuming the parameters are known and that $X_{t}=0$ for $t \leq 0$, the $h$ steps ahead forecast, $X_{n}(h)$, that minimizes the prediction mean square error, is given by

$$
\mathrm{X}_{\mathrm{n}}(\mathrm{h})=\sum^{\infty} \varphi_{\mathrm{j}}\left(\left[\mathrm{X}_{\mathrm{n}+\mathrm{h}-\mathrm{j}}\right]-\mu\right)+\sum^{\mathrm{q}} \theta_{\mathrm{j}}\left[\epsilon_{\mathrm{n}+\mathrm{h}-\mathrm{j}}\right]+\mu, \quad \mathrm{h}>1,
$$

where [.] denotes the conditional expectation of $=$ the variable given the $=1$ observations up to time $n$ and $\varphi(B)=$ $\phi(B)(1-B)^{\mathrm{d}}$.

Prediction intervals in long memory can be calculated using the same methodology employed in the classical prediction intervals for autoregression (see Box and Jenkins(1976)). Assuming that the distribution of $\epsilon_{t}$, $F_{\epsilon}$, is Gaussian with zero mean and variance $\sigma_{\epsilon}^{2}$, the usual $100(1-2 \alpha) \%$ level prediction interval for $\mathrm{X}_{\mathrm{n}+\mathrm{h}}$ is given by

$$
\left[\widehat{X}_{\mathrm{n}}(\mathrm{h})-\mathrm{z}_{1-\alpha} \widehat{\sigma}_{\epsilon} \widehat{\mathrm{V}}(\mathrm{h}), \quad \widehat{\mathrm{X}}_{\mathrm{n}}(\mathrm{h})+\mathrm{z}_{1-\alpha} \widehat{\sigma}_{\epsilon} \widehat{\mathrm{V}}(\mathrm{h})\right]
$$

where $\widehat{X}_{n}(h)$ is the prediction given in Equation (4) with the parameters replaced by their estimated values, $\mathrm{z}_{\alpha}$ is the $\alpha$ quantile of a standard normal variable, $\widehat{\sigma}_{\epsilon}$ is an estimate of $\sigma_{\epsilon}$ and $\widehat{V}(\mathrm{~h})=.11+\widehat{\psi}_{1}^{2}+\ldots+\widehat{\psi}_{\mathrm{h}-1}^{2}$, with $\widehat{\psi}_{\mathrm{i}}$ indicating the estimated parameters of the infinite-order moving average representation in (1), obtained by solving the following equation, $\left(1+\psi_{1} B+\psi_{2} B^{2}+\ldots\right)=\phi(B)^{-1}(1-B)^{-d} \theta(B)$.

The problem with the classical interval is that, if the necessary assumptions of the Box\& Jenkins methodology (known parameters and Gaussian distribution) are not satisfied, the actual coverage of the intervals in (5) can be different from the nominal level adopted. By taking into account parameter estimation variability and making no assumption on the errors distribution, bootstrap intervals may overcome this problem.

\section{Bootstrap prediction intervals}

As stated in Section 2, two sources of errors can arise from the asymptotic prediction interval given in Equation (5). The first one is that there is a parametric assumption about $F_{\epsilon}$, namely the Gaussian distribution, which may not be true for real data. The second problem with the asymptotic interval is that the sampling variability of the parameter estimators is not taken into account, which can lead to lower-than-nominal coverage intervals (Pascual et al, 2004).

For the ARFIMA model defined in (1), after properly estimating the parameters $\phi, \theta$ and $\mathrm{d}$, the estimated residuals, $\hat{\epsilon}_{t}$, are calculated as

$$
\hat{\epsilon}_{t}=\theta^{-1}(B) \widehat{\phi}(B)(1-B)^{\hat{d}}\left(X_{t}-\hat{\mu}\right), \quad t=1, \ldots, n
$$


where $\hat{\mu}$ is the sample mean. $\hat{\epsilon}_{\mathrm{t}}$ are supposed to be uncorrelated if the model is correctly specified.

After centering and rescaling the residuals, the nonparametric bootstrap consists of resampling these residuals with replacement so that the bootstrap series $X_{t}^{*}$ can be constructed.A typical bootstrap replicate in autoregressive models is generally obtained by fixing the first or the last values of the bootstrap series as the original values.Some authors, such as Thombs and Schucany (1990), work with the backward representation and fix the last $\mathrm{p}$ observed values of $\mathrm{X}_{\mathrm{t}}$, while some other works, as the one in Pascual et al. (2004), fix the first $\mathrm{p}+\mathrm{dvalues}$ as the original set.

In the ARFIMA model, the term $(1-B)$ dis calculated using the binomial expansion,

$$
(1-B)^{\mathrm{d}}=\sum_{\mathrm{j}=0}^{\infty} \frac{\Gamma(\mathrm{j}-\mathrm{d})}{\Gamma(\mathrm{j}+1) \Gamma(-\mathrm{d})},
$$

which implies an infinite recursion in order to obtain the residuals. This intrinsic characteristic of the ARFIMA model requests a truncation on some past value when performing the recursions to build the bootstrap series. Let $K$ be the truncation time. In this work, instead of fixing a set of Kinitial values of the variable $X_{t}$, say $\left\{X_{1}, \ldots, X_{K}\right\}$, the bootstrap series $X_{1}^{*}, X_{2}^{*}, \ldots X_{n}^{*}$ will be recursively generated in the following manner. For a time series of size $n, n+K$ values $\mathrm{Z}_{1}^{*}, \mathrm{Z}_{2}^{*}, \ldots, \mathrm{Z}_{\mathrm{n}+\mathrm{K}}^{*}$ are calculated as,

$$
\begin{aligned}
Z_{1}^{*} & =\widehat{\mu}+\epsilon_{1}^{*}, \\
Z_{2}^{*} & =\widehat{\varphi}_{1} \widetilde{Z}_{1}^{*}+\widehat{\theta}_{1} \epsilon_{1}^{*}+\hat{\mu}+\epsilon_{2}^{*}, \\
& \cdots, \\
Z_{n+K}^{*} & =\sum_{j=1}^{n+K-1} \widehat{\varphi}_{j} \widetilde{Z}_{n+K-j}^{*}+\sum_{j=1}^{q} \widehat{\theta}_{j} \epsilon_{n+K-j}^{*}+\widehat{\mu}+\epsilon_{n+K^{\prime}}^{*}
\end{aligned}
$$

where $\widetilde{Z}_{t}^{*}=Z_{t}^{*}-\widehat{\mu}, \widehat{\varphi}_{1}, \widehat{\varphi}_{2}, \ldots$ are the coefficients of the polynomial $\widehat{\varphi}(B)=\widehat{\phi}(B)(1-B)^{\widehat{d}}$ and $\epsilon_{t}^{*}, t=1, \ldots, n+K$ are random draws from $\widehat{F}(\epsilon)$, the empirical distribution function of the centered residuals. The bootstrap series, $X_{t}^{*}$, is then defined as

$$
X_{t}^{*}=Z_{t+K}^{*}, \quad t=1, \ldots, n
$$

$K$ should be large enough so that the initial values of $X_{t}^{*}$ mimic well enough those of a long memory time series. The algorithm described here only makes sense if the parameters estimated from the original series are those of a stationary and invertible model.

Thus, the proposed method of generating the nonparametric bootstrap replicates circumvents the need of fixing first or last observations in order to calculate the bootstrap series.

In what follows, two bootstrap prediction intervals for ARFIMA models are proposed and they will be based on the bootstrap series defined in (6). Such intervals, though, can work for any method of generating bootstrap series. The first interval employs the percentile method to calculate the quantiles of the bootstrap distribution function of $X_{n+h}^{*}$. The second procedure is based on the method proposed by Masarotto (1990) for pure autoregressive models and is adapted here to the ARFIMA model.

\subsection{PBAP interval}

The percentile bootstrap ARFIMA prediction (PBAP) interval, which to the knowledge of the authors were never used to ARFIMA models consists of taking the $\alpha$ and $(1-\alpha)$ percentiles of the bootstrap distribution of $X_{n+h}^{*}$. In practice, the procedure consists of the following steps:

1. Generate $B$ bootstrap series of size $\mathrm{n}$, as defined in (6).

2. Estimate the parameters $\mu^{*}, \varphi_{1}^{*}, \ldots, \varphi_{p}^{*}, \theta_{1}^{*}, \ldots, \theta_{q}^{*}$ and $d^{*}$, for each bootstrap series.

3. For each bootstrap series, a $\mathrm{h}$ steps ahead bootstrap future value is calculated recursively as

$$
X_{n+h}^{*}=\sum_{j=1}^{n-1} \varphi_{j}^{*}\left(\left[X_{n+h-j}^{*}\right]-\mu^{*}\right)+\sum_{j=1}^{q} \theta_{j}^{*}\left[\epsilon_{n+h-j}^{*}\right]+\mu^{*}+\epsilon_{n+h^{\prime}}^{*}
$$

where

$$
\left[X_{n+h-j}^{*}\right]= \begin{cases}X_{n+h-j}^{*}, & \text { if } h-j>0 \\ X_{n+h-j}, & \text { if } h-j \leq 0\end{cases}
$$


and $\epsilon_{n+h-j}^{*}, j<h$ are random draws from $\widehat{F}(\epsilon)$.

$$
\left[\epsilon_{n+h-j}^{*}\right]= \begin{cases}\epsilon_{n+h-j}^{*}, & \text { if } h-j>0 \\ \hat{\epsilon}_{n+h-j}, & \text { if } h-j \leq 0\end{cases}
$$

4. The bootstrap future values, $X_{n+h^{*}}^{* 1}, X_{n+h}^{* B}$, are ordered and the $100(1-2 \alpha) \%$ percentile interval is then defined as

$$
\left[X_{n+h}^{*(\alpha)} ; \quad X_{n+h}^{*(1-\alpha)}\right]
$$

Thombs and Schucany (1990) prove the large-sample validity of the above interval based on the limiting behavior of the bootstrap distribution of $X_{n+h}^{*}$, for $\operatorname{AR}(p)$.

\subsection{BPE interval}

The idea of the bootstrap prediction error (BPE) interval, based on the interval proposed by Masarotto (1990) and used for ARFIMA models in Bisaglia and Grigoletto (2001), is to calculate the empirical distribution of the bootstrap prediction errors and use the quantiles of this distribution to build the prediction interval.

For autoregressive series, Masarotto (1990) claims that, to build prediction intervals for $X_{n+h}$, the distribution of the standardized prediction errors

$$
r_{n, h}=\frac{X_{n+h}-\widehat{X}_{n}(h)}{\sigma_{\epsilon} V(h)},
$$

where $\sigma_{\epsilon}$ is the standard error of $\epsilon_{t}$ and $V(h)={ }_{1} \mid 1+\psi_{1}^{2}+\ldots+\psi_{h-1}^{2}$, with $\psi_{i}$ indicating the parameters of the infinite-order moving average representation of (1), should be approximated by the bootstrap empirical distribution of $r_{n, h}$. The author performed some Monte Carlo experiments to show the effectiveness of the proposed bootstrap approach using standardized prediction errors.

After estimating the parameters of the original series, the construction of the BPE interval with confidence level $100(1-2 \alpha) \%$ for $X_{n+h}$ follows the steps:

1. Generate $\mathrm{B}$ bootstrap series of size $\mathrm{n}+\mathrm{h}, X_{1}^{*}, X_{2}^{*}, \ldots, X_{n+h}^{*}$, as defined in (6).

2. Estimate the parameters $\mu^{*}, \varphi_{1}^{*}, \ldots, \varphi_{p}^{*}, \theta_{1}^{*}, \ldots, \theta_{q}^{*}$ and $d^{*}$, for each bootstrap series, using the first $n$ values $, X_{1}^{*}, \ldots, X_{n}^{*}$.

3. Calculate, for each bootstrap series, the bootstrap predicted value $\mathrm{h}$ steps ahead, $X_{n}^{*}(h)$, such as in (4), replacing the original series by the bootstrap series and the original parameters by the parameters estimated from the bootstrap series: $\mu^{*}, \varphi_{1}^{*}, \ldots, \varphi_{p}^{*}, \theta_{1}^{*}, \ldots, \theta_{q}^{*}$ and $d^{*}$.

4. Calculate the standardized bootstrap prediction error,

$$
\hat{r}_{n, h}^{*}=\frac{X_{n}^{*}(h)-X_{n+h}^{*}}{\widehat{\sigma}_{\epsilon}^{*} \widehat{V}^{*}(h)}
$$

5. Sort the B bootstrap standardized prediction errors and let $\widehat{r}_{n, h}^{*(j)}$ be the $j$-th bootstrap prediction error. The $100(1-2 \alpha) \% \mathrm{BPE}$ interval is then calculated as

$$
\left[\widehat{X}_{n}(h)+\widehat{r}_{n, h}^{*(\alpha)} \widehat{\sigma}_{\epsilon} \widehat{V}(h) ; \quad \widehat{X}_{n}(h)+\widehat{r}_{n, h}^{*(1-\alpha)} \widehat{\sigma}_{\epsilon} \widehat{V}(h)\right]
$$

\section{Empirical results}

Monte Carlo experiments were performed to compare the bootstrap prediction intervals. of Section 3 (PBAP and BPE) with the asymptotic (Asymp) interval. The experiments were carried out for four different models: Model 1: ARFIMA $(0,0.3,0)$ with Normal errors; Model 2: $\operatorname{ARFIMA}(1,0.3,1)$ with Normal errors;Model 3: ARFIMA(1,0.3,1) with Exponential errors and Model 4: $\operatorname{ARFIMA}(1,0.3,1)$ with a mixture distribution for the errors.In all cases parameters $\phi$ and $\theta$ were fixed at 0.3 and the errors were standardized to have zero mean and unit variance. In the processes with mixture errors, there is a probability of 0.9 that the errors come from a Normal distribution with mean -1 andvariance 1 and a probability of 0.1 that the errors come from a Normal distribution with mean 9 and variance 1.Two lead times ( $h=1$ and 10) were adopted and the estimators used for $d$ were the GPH and FT.

For each model, 1000 Monte Carlo experiments were performed with the specified parameters and, for each Monte Carlo, 999bootstrap series were generated. A nominal coverage for the prediction intervals of $95 \%$ and a sample size equal to 100 were used in all simulations. Tables present the average coverage rates, length of the intervals and the 
average one-tail error rates below and above. In order to better compare the intervals in the case of asymmetric errors, the sum of the absolute differences between the theoretical one-tail errors and actual errors were calculated. In the tables that follow such sum is represented by the letter $\mathrm{S}$.

Table 1 shows the results for an $\operatorname{ARFIMA}(0,0.3,0)$ with Normal errors. This is a relatively good condition for the asymptotic interval: though the sample size is not large, there are few parameters to be estimated and the errors are normally distributed. Even so, under these ideal conditions, the bootstrap intervals were at least as good as the asymptotic one. The BPE interval, in particular, seemed to have the closest coverage rate to the nominal level assumed.

Table 2 shows the results for an $\operatorname{ARFIMA}(1,0.3,1)$ with Normal errors. Even though the errors are normally distributed, now there are more parameters to be estimated, which makes the estimation less accurate and thus reduces, in general, the actual coverage of the asymptotic interval. In this case the bootstrap intervals get closer to the theoretical coverage level of $95 \%$. The non-coverage in the tails are also closer to the expected $2.5 \%$ for the bootstrap intervals.

\begin{tabular}{|c|c|c|c|c|c|c|}
\hline $\begin{array}{l}\text { Lead } \\
\text { time }\end{array}$ & Method & Interval & $\begin{array}{l}\text { Average } \\
\text { Coverage }\end{array}$ & $\begin{array}{l}\text { Coverage } \\
\text { below/above }\end{array}$ & $S$ & $\begin{array}{l}\text { Average } \\
\text { Length }\end{array}$ \\
\hline \multirow{7}{*}{1} & & Asymp & 94.41 & $2.77 / 2.82$ & 0.59 & 3.90 \\
\hline & FT & $P B A P$ & 94.47 & $2.81 / 2.73$ & 0.54 & 3.98 \\
\hline & & $B P E$ & 94.84 & $2.59 / 2.57$ & 0.16 & 4.04 \\
\hline & & & & & & \\
\hline & & Asymp & 94.20 & $2.89 / 2.91$ & 0.80 & 3.94 \\
\hline & GPH & $P B A P$ & 94.14 & $3.04 / 2.82$ & 0.86 & 4.16 \\
\hline & & $B P E$ & 94.66 & $2.70 / 2.65$ & 0.35 & 4.09 \\
\hline \multirow{7}{*}{10} & & Asymp & 94.48 & $2.71 / 2.81$ & 0.52 & 4.34 \\
\hline & FT & $P B A P$ & 95.18 & $2.47 / 2.35$ & 0.18 & 4.49 \\
\hline & & $B P E$ & 94.92 & $2.51 / 2.57$ & 0.08 & 4.49 \\
\hline & & & & & & \\
\hline & & Asymp & 93.14 & $3.38 / 3.48$ & 1.86 & 4.09 \\
\hline & GPH & $P B A P$ & 93.02 & $3.58 / 3.40$ & 1.98 & 4.14 \\
\hline & & $B P E$ & 93.87 & $3.07 / 3.06$ & 1.13 & 4.28 \\
\hline
\end{tabular}

Table 1. Confidence interval rates (in percentage) for the $\operatorname{ARFIMA}(0, d, 0)$ model, with $d=0.3$ and Normal errors

\begin{tabular}{|c|c|c|c|c|c|c|}
\hline $\begin{array}{l}\text { Lead } \\
\text { time }\end{array}$ & Method & Interval & $\begin{array}{l}\text { Average } \\
\text { Coverage }\end{array}$ & $\begin{array}{l}\text { Coverage } \\
\text { below/above }\end{array}$ & $S$ & $\begin{array}{l}\text { Average } \\
\text { Length }\end{array}$ \\
\hline \multirow{7}{*}{1} & & Asymp & 93.89 & $3.04 / 3.08$ & 1.12 & 3.87 \\
\hline & FT & $P B A P$ & 95.22 & $2.48 / 2.29$ & 0.23 & 4.45 \\
\hline & & $B P E$ & 94.69 & $2.64 / 2.67$ & 0.31 & 4.06 \\
\hline & & & & & & \\
\hline & & Asymp & 94.07 & $2.97 / 2.96$ & 0.93 & 3.88 \\
\hline & GPH & $P B A P$ & 95.68 & $2.22 / 2.10$ & 0.68 & 4.36 \\
\hline & & $B P E$ & 94.74 & $2.63 / 2.63$ & 0.26 & 4.05 \\
\hline \multirow{7}{*}{10} & & Asymp & 92.88 & $3.51 / 3.61$ & 2.12 & 6.05 \\
\hline & FT & $P B A P$ & 95.14 & $2.52 / 2.33$ & 0.19 & 6.81 \\
\hline & & $B P E$ & 94.52 & $2.69 / 2.79$ & 0.48 & 6.61 \\
\hline & & & & & & \\
\hline & & Asymp & 93.75 & $3.14 / 3.11$ & 1.25 & 6.27 \\
\hline & GPH & $P B A P$ & 96.35 & $1.84 / 1.80$ & 1.36 & 7.04 \\
\hline & & $B P E$ & 95.00 & $2.52 / 2.47$ & 0.05 & 6.79 \\
\hline
\end{tabular}

Table 2. Confidence interval rates (in percentage) for the ARFIMA $(1, d, 1)$ model, with $\phi=0.3, \theta=0.3, d=0.3$ and Normal errors

Table 3 and Table 4 show the results of two ARFIMA(1,0.3,1) models with respectively exponential and mixture errors. In these cases the asymptotic intervals clearly underperform. In the case of exponential errors the asymptotic interval does come relatively close to 0.95 , but the non-coverages below and above are very different from what they were supposed to be. The bootstrap intervals manage a much more symmetric behavior for the intervals, which can be confirmed by the smallest values of $\mathrm{S}$.

It is worth noting in Table 2, Table 3 and Table 4 that, even though the PBAP and BPE intervals had a somewhat similar performance in terms of average coverage, the BPE interval consistently presented smaller size.

The code of Monte Carlo simulations described above were written for Fortran 77. None of them took more than 3 hours and a half to be completed, with no more than an average of 13 seconds for each of the 1000 Monte Carlo replication. 


\begin{tabular}{|c|c|c|c|c|c|c|}
\hline $\begin{array}{l}\text { Lead } \\
\text { time }\end{array}$ & Method & Interval & $\begin{array}{l}\text { Average } \\
\text { Coverage }\end{array}$ & $\begin{array}{l}\text { Coverage } \\
\text { below/above }\end{array}$ & $S$ & $\begin{array}{l}\text { Average } \\
\text { Length }\end{array}$ \\
\hline \multirow{7}{*}{1} & & Asymp & 94.12 & $0.00 / 5.88$ & 5.88 & 3.89 \\
\hline & FT & $P B A P$ & 96.12 & $1.09 / 2.78$ & 1.69 & 4.56 \\
\hline & & $B P E$ & 96.59 & $0.68 / 2.73$ & 2.05 & 4.11 \\
\hline & & & & & & \\
\hline & & Asymp & 94.33 & $0.03 / 5.64$ & 5.61 & 3.90 \\
\hline & GPH & $P B A P$ & 96.60 & $0.92 / 2.48$ & 1.60 & 4.46 \\
\hline & & $B P E$ & 96.64 & $0.65 / 2.70$ & 2.05 & 4.08 \\
\hline \multirow{7}{*}{10} & & Asymp & 93.73 & $0.80 / 5.47$ & 4.67 & 6.10 \\
\hline & FT & $P B A P$ & 95.39 & $1.86 / 2.75$ & 0.89 & 6.94 \\
\hline & & $B P E$ & 94.38 & $2.82 / 2.80$ & 0.62 & 6.71 \\
\hline & & & & & & \\
\hline & & Asymp & 94.24 & $0.62 / 5.14$ & 4.52 & 6.28 \\
\hline & GPH & $P B A P$ & 96.37 & $1.31 / 2.32$ & 1.37 & 7.12 \\
\hline & & $B P E$ & 94.93 & $2.41 / 2.66$ & 0.25 & 6.89 \\
\hline
\end{tabular}

Table 3. Confidence interval rates (in percentage) for the $\operatorname{ARFIMA}(1, d, 1)$ model, with $\phi=0.3, \theta=0.3, d=0.3$ and Exponential errors

\begin{tabular}{|c|c|c|c|c|c|c|}
\hline $\begin{array}{l}\text { Lead } \\
\text { time }\end{array}$ & Method & Interval & $\begin{array}{l}\text { Average } \\
\text { Coverage }\end{array}$ & $\begin{array}{l}\text { Coverage } \\
\text { below/above }\end{array}$ & $S$ & $\begin{array}{l}\text { Average } \\
\text { Length }\end{array}$ \\
\hline \multirow{7}{*}{1} & & Asymp & 90.25 & $0.00 / 9.74$ & 9.74 & 3.85 \\
\hline & FT & $P B A P$ & 95.08 & $1.67 / 3.26$ & 1.59 & 4.61 \\
\hline & & $B P E$ & 96.48 & $1.30 / 2.22$ & 1.48 & 4.35 \\
\hline & & & & & & \\
\hline & & Asymp & 90.28 & $0.04 / 9.67$ & 9.63 & 3.84 \\
\hline & GPH & $P B A P$ & 95.96 & $1.36 / 2.69$ & 1.33 & 4.52 \\
\hline & & $B P E$ & 96.54 & $1.37 / 2.09$ & 1.54 & 4.31 \\
\hline \multirow{7}{*}{10} & & Asymp & 93.18 & $0.19 / 6.63$ & 6.44 & 6.04 \\
\hline & FT & $P B A P$ & 95.59 & $1.81 / 2.60$ & 0.79 & 6.72 \\
\hline & & $B P E$ & 94.39 & $2.99 / 2.62$ & 0.61 & 6.47 \\
\hline & & & & & & \\
\hline & & Asymp & 93.74 & $0.14 / 6.12$ & 5.98 & 6.23 \\
\hline & GPH & $P B A P$ & 96.56 & $1.27 / 2.16$ & 1.57 & 6.88 \\
\hline & & $B P E$ & 95.24 & $2.45 / 2.31$ & 0.24 & 6.71 \\
\hline
\end{tabular}

Table 4. Confidence interval rates (in percentage) for the ARFIMA $(1, d, 1)$ model, with $\phi=0.3, \theta=0.3, d=0.3$ and Mixture errors

\section{An application to real data}

The methods presented in this paper were applied to a real data set. The data used was the average annual temperature in New York city between 1886 and 2011 in decimals of Celsius. The data was extracted from the website of the National Climatic Data Center of the United States government. The values between 1886 and 2001 were used to fit the model while the remaining ones were used to compare the prediction intervals constructed. Initially, an $\operatorname{ARFIMA}(0, d, 0)$ model was fitted to the data because of the slow decay of the autocorrelation function (see Figure 1). Using the FT method the estimates were $\widehat{d}=0.3725$ and $\widehat{\sigma}=6.814$. The residuals of the fitted model seemed to behave as white noise (see Figure 2). A Ljung-Box test applied to the residuals did not reject the white noise hypothesis with a p-value of 0.1823 , thus the $\operatorname{ARFIMA}(0, d, 0)$ model was considered appropriate for the data. The normality assumption for the residuals was rejected by the Kolmogorov-Smirnov test with a p-value below 0.0001 .

Figure 3 shows the graph of the series starting from 1972 and the prediction intervals from 2002 to 2011 (1 to 10 steps ahead). This is a case in which there are only two parameters to be estimated, the mean and $d$, even so the bootstrap intervals slightly differ from the asymptotic interval. The asymptotic interval presents smaller widths but, as it was seen in the simulations, it does not capture all the variability present in the predictions.

\section{Conclusions}

In this work, two bootstrap prediction intervals, which take into account the uncertainty associated to the parameter estimation,are tested for the ARFIMA model. The resampled series is constructed using the infinite autoregressive form of the ARFIMA model, thus avoiding the restriction of fixing initial of final known values for the bootstrap series. The bootstrap methods were empirically compared to the standard asymptotic prediction interval,based on the Gaussian assumption. An extensive Monte Carlo study was conducted assuming Gaussian and non-Gaussian distribution for the errors. The results confirmed the good performance of the bootstrap intervals with respect to the coverage rate, especially in the non-Gaussian case. The BPE intervals seems to have slightly better performance than the PBAP 
interval.

\section{Acknowledgements}

The authors gratefully acknowledge partial financial support from Fapes/ES, Fapemig/MG and CNPq/Brazil and CentraleSupélec, Paris, France.

\section{References}

1. Bhansali RJ, Kokosza PS (2002) Computation of the forecast coecients for multistep prediction of long-range dependent time series. International Journal of Forecasting 18: 181-206.

2. Bisaglia L, Grigoletto M (2001) Prediction intervals for farima processes bybootstrap methods. Journal of Statistical Computation and Simulation 68:185-201.

3. Boutahar M (2007) Optimal prediction with nonstationary ARFIMA model. Journal of Forecasting. 26: 95-111.

4. Box GEP, Jenkins GM (1976) Time Series Analysis: Forecasting and Control, 2nd ed. Holden Day: San Francisco. Crato N, Ray BK (1996) Model selection and forecasting for long-range dependent processes. Journal of Forecasting 15: 107 - 125.

5. Doukham P, Oppenheim G, Taqqu MS (2002) Theory and Applications of Long-Range Dependence. Birkhauser, Boston. Fox R, Taqqu MS (1986) Large-sample properties of parameter estimates for strongly dependent stationary Gaussian time series. The Annals of Statistics 14: 517-532.

6. Franco GC, Reisen VA (2007) Bootstrap approaches and condence intervals for stationary and non-stationary long-range dependence processes. Physica A 375: 546-562.

7. Geweke J, Porter-Hudak S (1983) The estimation and application of long memory time series model. Journal of Time Series Analysis 4: 221-238.

8. Granger CWJ, Joyeux R (1980) An introduction to long-memory time series models and fractional dierencing. Journal of Time Series Analysis 1: 15-29.

9. Hosking J (1981) Fractional dierencing. Biometrika 68: 165-176.

10. Masarotto G (1990) Bootstrap prediction intervals for autoregressions. International Journal of Forecasting 6: 229-239.

11. Pan L, Politis DN (2016) Bootstrap prediction intervals for linear, nonlinear and nonparametric autoregressions. Journal of Statistical Planning and Inference 177: 1-27.

12. Pascual L, Romo J, Ruiz E (2004) Bootstrap predictive inference for ARIMA processes. Journal of Time Series Analysis 25: 449-465.

13. Pfeermann D, Tiller R (2005) Bootstrap approximation to prediction MSE for state-space models with estimated parameters. Journal of Time Series Analysis 26: 893-916.

14. Rodriguez A, Ruiz E (2010) Bootstrap prediction intervals in state space models. Journal of Time Series Analysis 30: 167-178.

15. Rupasinghe M, Mukhopadhyay P, Samaranayake, VA (2013) Obtaining prediction intervals for FARIMA processes using the sieve bootstrap. Journal of Statistical Computation and Simulation 84: 2044-2058.

16. Rupasinghe M, Samaranayake VA (2012) Asymptotic properties of sieve bootstrap prediction intervals for FARIMA processes. Statistics and Probability Letters 82: 2108-2114.

17. Thombs LA, Schucany WR (1990) Bootstrap prediction intervals for autoregression. Journal of the American Statistical Association 85: 486-492.

18. Wall K D, Stoer DA (2002) State space approach to bootstrapping conditional forecasts in ARMA models. Journal of Time Series Analysis 23:733-752. 